\title{
INJECTION CHARACTERISTICS OF NON-SWIRLING AND SWIRLING ANNULAR LIQUID SHEETS
}

\author{
E. A. Ibrahim and T. R. McKinney \\ Mechanical Engineering Department \\ Tuskegee University \\ Tuskegee, Alabama
}

\begin{abstract}
A simplified mathematical model, based on body-fitted coordinates, is formulated to study the evolution of non-swirling and swirling liquid sheet emanated from an annular nozzle in a quiescent surrounding medium. The model provides predictions of sheet trajectory, thickness and velocity at various liquid mass flow rates and liquid-swirler angles. It is found that a non-swirling annular sheet converges toward its centerline and assumes a bell shape as it moves downstream from the nozzle. The bell radius, and length are more pronounced at higher liquid mass flow rates. The thickness of the non-swirling annular sheet increases while its stream-wise velocity decreases with an increase in mass flow rate. The introduction of swirl results in the formation of a diverging hollow-cone sheet. The hollow-cone divergence from its centerline is enhanced by an increase in liquid mass flow rate or liquid-swirler angle. The hollowcone sheet its radius, curvature and stream-wise velocity increase while its thickness and tangential velocity decrease as a result of increasing the mass flow rate or liquid-swirler angle. The present results are compared with previous studies and conclusions are drawn.
\end{abstract}

\section{INTRODUCTION}

Combustion efficiency and stability are predominated by fue!/oxidizer injection and atomization. Hence, it is imperative to optimize the injector design and combustor aerodynamics to achieve rapid and uniform fuel/oxidizer mixing. The swirl atomizer is particularly suited to high-thrust and high-performance liquid-propellant rocket engines where intimate mixing of reactants is essential to achieving a uniform distribution of mixture ratios in the combustion chamber. The distribution of the mixture ratios and mass fluxes needs to ensure not only maximum performance but also adequate cooling of the hardware. Recent experimental studies ${ }^{1,2}$ have revealed that apart from enhancing atomization, swirl atomizers promote combustion stability.

Current designs of swirl atomizers depend on extensive experimentation to arrive at satisfactory results in terms of combustion efficiency and stability. ${ }^{3}$ Such experiments are expensive and time consuming given the vast number of parameter variations involved. Current CFD combustion simulation codes rely heavily on empirical atomization characteristics as initial conditions. Therefore, it is highly desirable to develop advanced injection and atomization models to be incorporated in combustion simulation codes that could be used to guide the optimization of injector design.

Many swirl atomizer models $\mathbf{s}^{3.4}$ have been based on linearized perturbation analyses of a plane liquid sheet. ${ }^{5,6,7}$ The framework developed by Dombrowski and Johns ${ }^{6}$ constitutes the foundation for the bulk of these models. Dombrowski and Johns ${ }^{6}$ hypothesized that sheet breakup was due to the growth of Kelvin-Helmholtz instability waves. The sheet was assumed to disintegrate into ligaments with a diameter that depended on the wavelength of dominant instability waves. The ligaments broke up into droplets according to Weber's ${ }^{8}$ analysis of the capillary instability. The breakup of the ligaments determined the mean drop size of the spray.

The breakup processes of a pre-filmed hollow-cone liquid sheet, such as that produced by air blast atomization, are similar to those of a plane sheet ${ }^{9,10}$ as described by Dombrowski and Johns ${ }^{6}$. However, the theory presented by Dombrowski and Johns ${ }^{6}$ does not include an account for the evolution of an annular sheet as it emerges from the nozzle. Dombrowski and Johns ${ }^{6}$ assumed that the sheet thickness is inversely proportional to distance from the nozzle and were able to determine the proportionality constant, $K$, for the fan spray nozzles they used in their experiments. Since $K$ is obtained empirically, it is not known a priori for a given nozzle and hence would represent an obstacle for a purely predictive model of sheet atomization. Recently, linearized perturbation analyses of non-swirling ${ }^{11}$ and swirling ${ }^{12}$ annular cylindrical jets have been

Approved for public release; distribution is unlimited.

"This work was performed under sponsorship of NASA Space Act Agreement NCC8-200. Our team thanks Brent Harper the COTR, and Gary Lyles and his staff for their technical inputs and encouragement. 
performed. However, these analyses are focused on predicting the most unstable wave number and maximum growth rates of the perturbation waves. The annular jet swirl is presumed to be of a free vortex type which is not realistic. Therefore, relations for determining sheet trajectory or flow parameters such as sheet thickness or velocity are not available through linearized perturbation studies.

Chuech and co-workers ${ }^{13-16}$ advanced a mathematical model that used curvilinear body-fitted coordinates to simplify the analysis of the evolution of non-swirling and swirling annular liquid sheets injected from a nozzle into a quiescent surrounding medium. They reported predictions of the spray angle, sheet thickness and velocities that were in general agreement with their experimental measurements. Although their model was published in several articles, accounts of the governing equations for the model were inconsistent. The details of the numerical technique they employed to solve the governing equations were not discussed in enough details in any of the numerous publications of the model to allow for independent verification. Despite that Chuech and co-workers' model contains a formulation for liquid-surrounding interfacial friction effects, the results they presented apply only to a sheet issued in a void, a fact that was not mentioned in their publications but has become evident through the course of the present work.

The purpose of the present work is to present a clear and accurate model to predict the spray characteristics of swirling and non-swirling annular sheets. These characteristics include, spray angle, radius, sheet thickness and velocity. The model presented constitutes a corrected version of that reported by Chuech and co-workers and comprises details of the solution technique to permit others to reproduce it without difficulty or guess work on their part. Since the particulars of the numerical solution method used by Chuech and co-workers ${ }^{13-16}$ were never reported, it wouldn't be possible to know if they were similar to the ones introduced here. The resilts presented in the present work differ irom those of Chuech and coworkers $^{13-16}$ in that the influence of surrounding medium is accounted for through the liquid-gas interfacial friction forces. Similar to Chuech and co-workers, non-swirling annular sheets are also included in the present study because of their important applications in spray atomization and to allow for the extraction of the effects of swirl on atomization by comparing and contrasting the behavior of non-swirling and swirling annular sheets. The work presented here should provide a basis for a comprehensive spray model to be implemented in CFD codes to simulate combustion performance. Currently such CFD codes neglect the presence of the hollow cone sheet and assume instantaneous atomization at the nozzle exit. However, it is well known ${ }^{17}$ that CFD predictions are very sensitive to the assumed atomization characteristics.

\section{MODEL FORMULATION}

A curvilinear coordinate system $\xi-\xi-\eta$, as shown in Fig. 1, is utilized as a non-inertial reference frame to analyze the liquid flow in a swirting axisymmetric hollow-cone sheet issued from a nozzle at an initial velocity $U_{0}$ and angle $\theta_{0}$ in a quiescent surrounding gas. The co-ordinates $\xi, \zeta, \eta$ are perpendicular to each other and coincide with the liquid streamwise, tangential, and normal to the streamline directions, respectively. The choice of a curvilinear coordinate system that conforms to the sheet boundaries simplifies the mathematical analysis because the normal velocity component vanishes. The liquid flow is assumed to be Newtonian, incompressible and inviscid. Since in practical spray applications, the sheet thickness is usually much smaller than the cone radius, variations of velocity and pressure across the film thickness may be neglected. Mathematically, the goveming equations describing conservation of mass and momentum per unit volume at steady state may be expressed as:

Continuity:

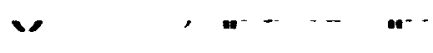

Momentum in the stream-wise $\xi$-direction:

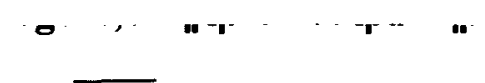

Momentum in the normal $\eta$-direction:

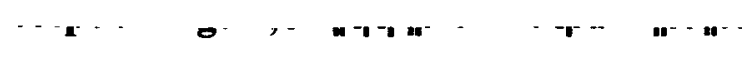


Momentum in the tangential $\zeta$-direction:

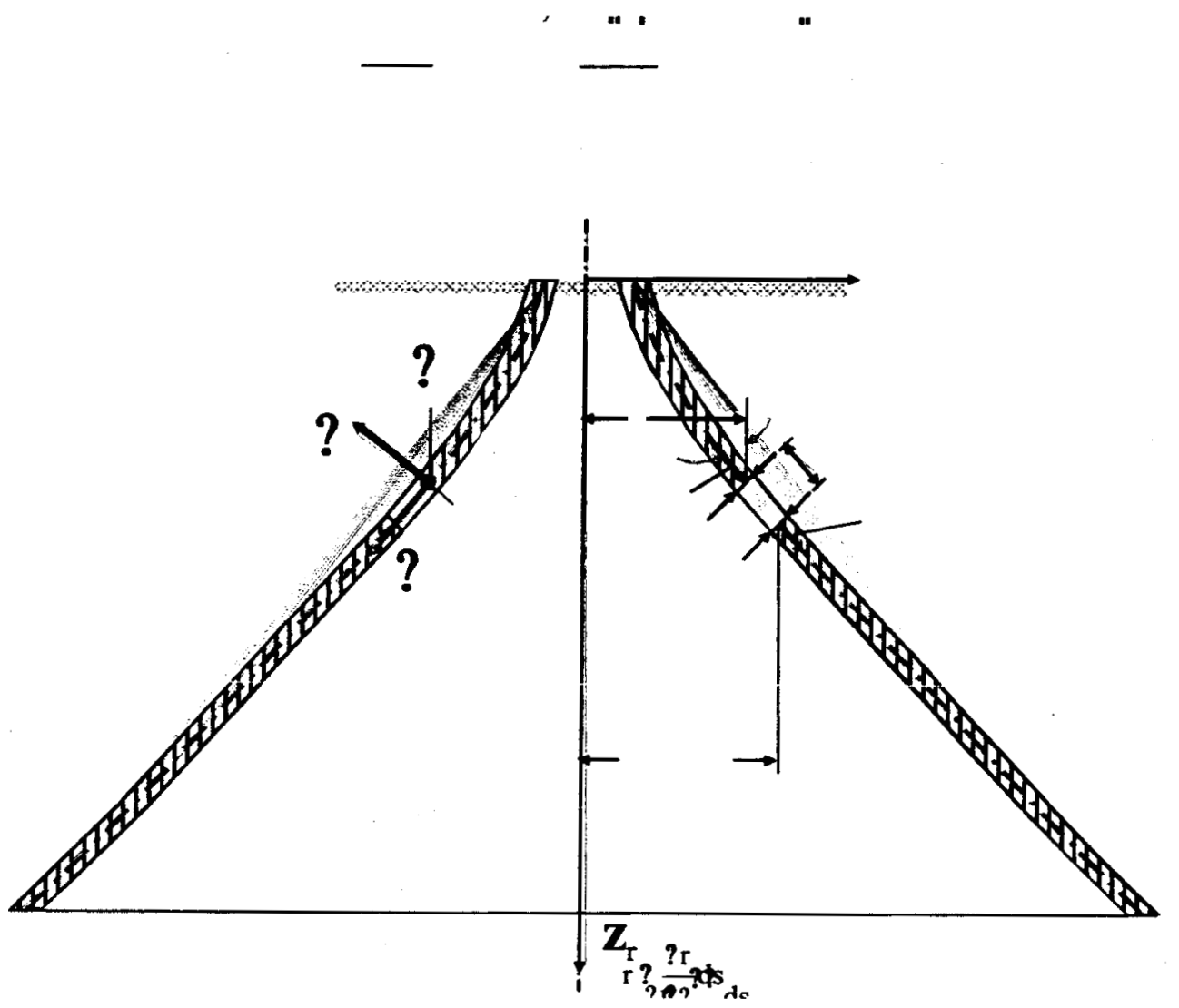

Figure 1. Schematic diagram of an annular liquid sheet.

Where $r$ stands for the radial coordinate of the reference frame and $\theta$ is the angle measured between the median streamline and axial direction $\mathrm{z}$ as shown in Fig. 1. S Shet refers to the sheet cross sectional area and $\delta$ is the local thickness of the annular liquid sheet. $p_{f}$ is liquid fuel pressure, $g$ is gravitational acceleration, $\rho_{f}$ is liquid density, $u_{f}$ and $w_{f}$ are the velocity components in the stream-wise and tangential directions, respectively. The first term in each of Eqs. (2) and (4) represent directional components of inertia forces in the stream-wise and tangential directions. The second term in each of Eqs. (2) and (4) and the first term in Eq. (3) denote directional components of centrifugal forces. The second term in Eq. 4 relates to Coriolis force. The terms $\rho_{f} g \cos \theta$ and $\rho_{f} g \sin \theta$ in Eqs. (2) and (3) designate directional components of gravity force. The terms $S_{\xi}$ and $S_{\zeta}$ in Eqs. (2), (3) account for the viscous forces in the stream- wise and tangential directions, respectively.

The pressure gradient in the normal direction $\eta$ can be approximated by its integrated form as a function of the gas pressure difference across the liquid gas interface and surface tension forces;

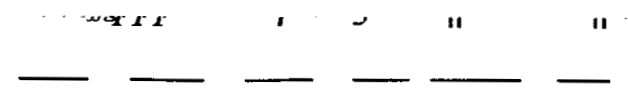

where $\Delta p_{\mathrm{f}}$ is the pressure differences across the liquid cross section of thickness $\delta$ in the normal direction, $\Delta p_{g}$ is the surrounding gas pressure difference across the sheet thickness, and $\sigma$ is liquid surface tension. 
Following Chuech and co-workers ${ }^{13-16}$, the viscous forces in the stream-wise and tangential momentum equations are accounted for through the interfacial friction forces acting on the inner and outer liquid-gas interfaces. Therefore, the viscous forces may be written, respectively, in terms of Rizk and Lefebvre's ${ }^{18}$ gasliquid interfacial friction factors representation as:

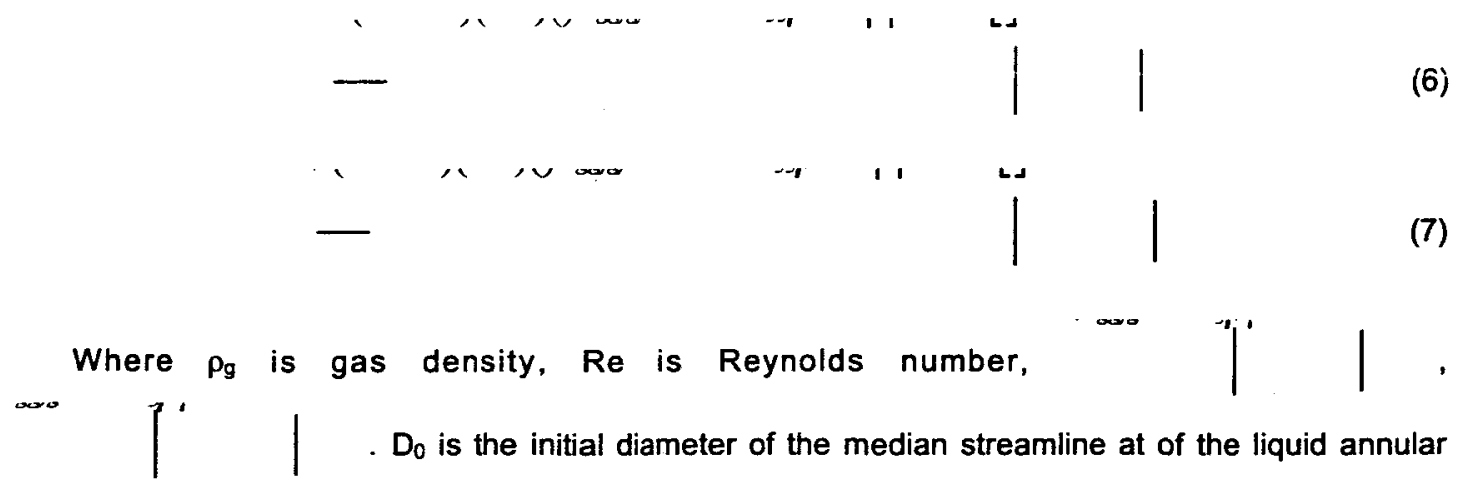

sheet at the nozzle exit, $u_{g}$ and $w_{g}$ are the respective stream-wise and tangential gas velocities, and $\mu_{g}$ is dynamic viscosity of the surrounding gas. The terms between the square brackets in Eqs. (16) and (17) designate Rizk and Lefebvre's ${ }^{18}$ interfacial friction factors.

As mentioned earlier, although Chuech and co-workers ${ }^{13-16}$ developed a similar mathematical model to the one presented above, their model's goveming momentum equations were reported in a conflicting manner in their published work. ${ }^{13-16}$ Therefore, the present model's equations represent a corrected and clear version of Chuech and co-workers model. The use of primitive variables in the present formulation is intended to add to the clarity of the model's equations.

Despite of the fact that Chuech and co-workers ${ }^{13-16}$ presented their hollow-cone spray model in numerous publications, details of the numerical solution method they used were grossly omitted. The obscurity of Chuech and co-workers ${ }^{13-16}$ solution technique renders any attempt to reconstruct their model open to speculation. The present work attempts to fill this gap by providing an informative but concise details of a numerical approach to obtain solutions for the model formulation presented.

Due to the simplifying assumptions and use of conforming curvilinear coordinates in the present model, all the dependent variables have gradient only in the stream-wise direction, $\xi$. Therefore, the governing equations given by Eqs. (1), (2), (3), (4) subject to Eqs. (5), (6), (7) may be simplified to a system of nonlinear first-order ordinary differential equations in the form

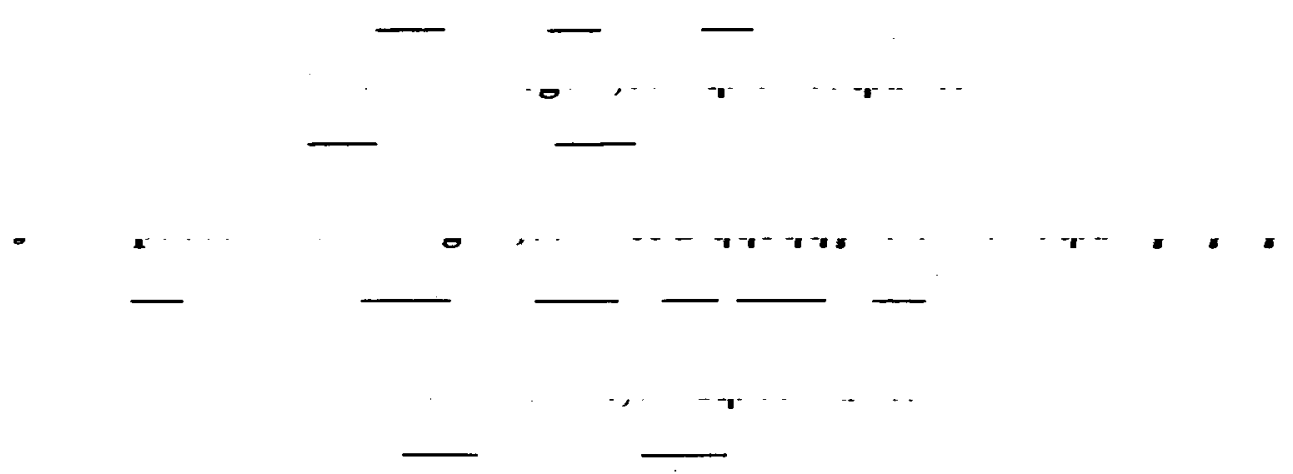

Since the system of equations (8), (9), (10), (11) subject to equations (6) and (7) include four equations and five unknowns, $r, \theta, \delta, u_{f}, w_{f}$ an additional equation is needed to make the system determinate. Such an equation may be derived from geometrical considerations of the median streamline as shown in Fig. 1. 
A set of five boundary conditions are needed to bring closure to the model. Since the hollow-cone liquid flow is bounded at nozzle orifice, the boundary conditions to be coupled with the system of ordinary differential equations may be stated as

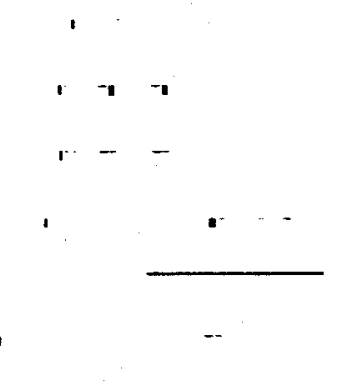

Where $\mathcal{R}_{0}$ and $\theta_{0}$ refer to the initial radius and angle of the median streamline at nozzle exit, respectively, $\delta_{0}$ is the initial thickness of the hollow-cone liquid sheet at nozzle exit, $f$ is the liquid fuel mass flow rate, $\alpha$ is the fuel-port angle or fuel-swirler angle, and $u_{f 0}, w_{f 0}$ are the respective initial velocity components of the hollow-cone liquid sheet fiow at nozzie exit in stream-wise and iangentiai directions.

To enable tracking of sheet trajectory, its axial coordinate $z$ is evaluated in reference with Fig. 1 as

subject to the boundary condition,

The system of nonlinear first-order ordinary differential equations given by (8), (9), (10), (11), (12), and (18) subject to the boundary conditions expressed as (13), (14), (15), (16), (17), and (19) is solved using a fifth order Runge-Kutta Verner method to yield solutions for $r, \theta, z, \delta, u_{f}, w_{f}$.

In the present model, since the surrounding gas is assumed to be quiescent, quantities representing gas velocity, $u_{\mathrm{g}}$ and $w_{\mathrm{g}}$, vanish from Eqs. (6) and (7). It is also assumed that the outer and inner gas pressures are equal so that, $\Delta p_{g}=0$. Note that results for a non-swirling annular sheet may be obtained when the liquid swir velocity, $W_{\mathrm{f}}$, is set to zero.

\section{RESULTS AND DISCUSSION}

\section{NON-SWIRLING SHEET}

Since a non-swirling hollow-cone sheet has its own practical applications in air assist and air-blast atomization ${ }^{11}$, we begin by studying this case by setting the swirl velocity, $w_{\mathrm{f}}$, to zero, which eliminates the effects of both the centrifugal and Coriolis forces from the present model. This study also allows for gaining insight into the differences in the development of non-swirling and swirling annular sheet and isolating effects that are solely due to swirl. To facilitate comparison with Baird and Davidson ${ }^{19}$ and Chuech and coworkers $^{13-16}$ results, the same nozzle geometry and flow conditions are used in the present investigation. Therefore, the injected sheet liquid is assumed to be water and the surrounding gas is atmospheric air. The initial mean sheet diameter $D_{0}=2 R_{0}$ at nozzle orifice is $12.41 \mathrm{~mm}$ and the initial water sheet thickness, $\delta_{0}$, is $0.29 \mathrm{~mm}$. The water sheet is assumed to be injected vertically downward so that initial sheet angle, $\theta_{0}$, is $0^{\circ}$. The liquid flow rates considered are $13.09,19.13,25.50$, and $31.88 \mathrm{~g} / \mathrm{s}$. The initial sheet velocity in the stream-wise direction, $u_{f 0}$, is calculated from the mass flow rates using Eq. (16). 
Predictions of dimensionless radius and angle of a non-swirling annular sheet with the axial distance from the nozzle are presented in Figs. 2 and 3. At a constant liquid flow rate, both the sheet radius and angle always decrease as the sheet moves away from the nozzle. Since the sheet angles are always negative, the absolute values of the angle are actually in the downstream direction. As a result, the annular sheet gradually contracts toward its centerline. The contraction of the sheet is due to surface tension forces. As the flow rate is increases, both sheet radius and angle are amplified due to greater inertia forces that counteract the contracting action of surface tension forces.

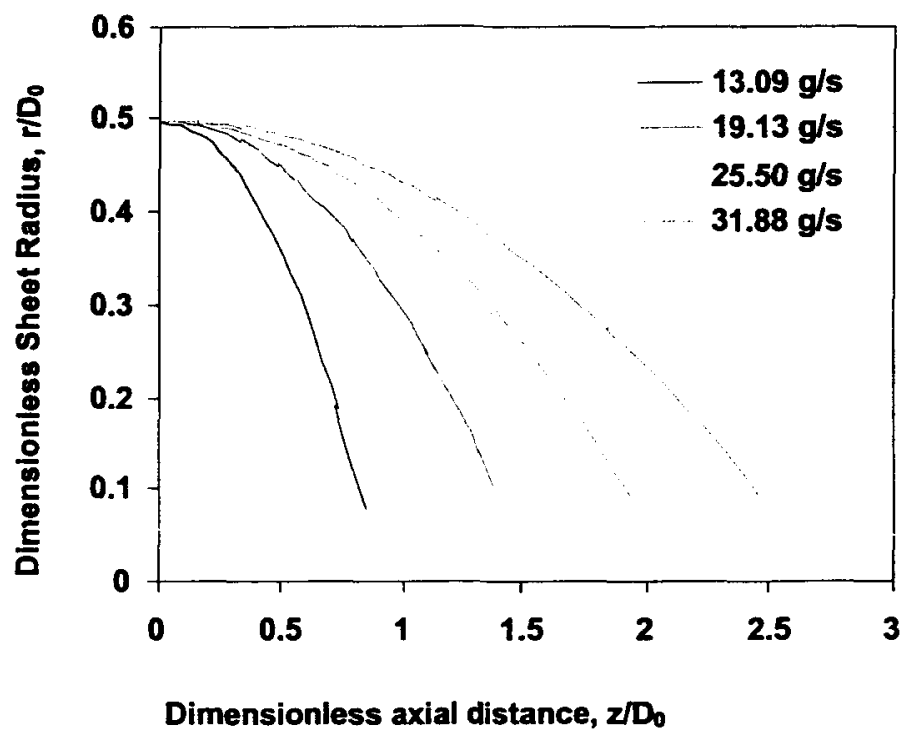

Figure 2. Axjal variation of dimensionless radius for a non-swirling liquid sheet.

Dimensionless axial distance, $z^{/ D_{0}}$

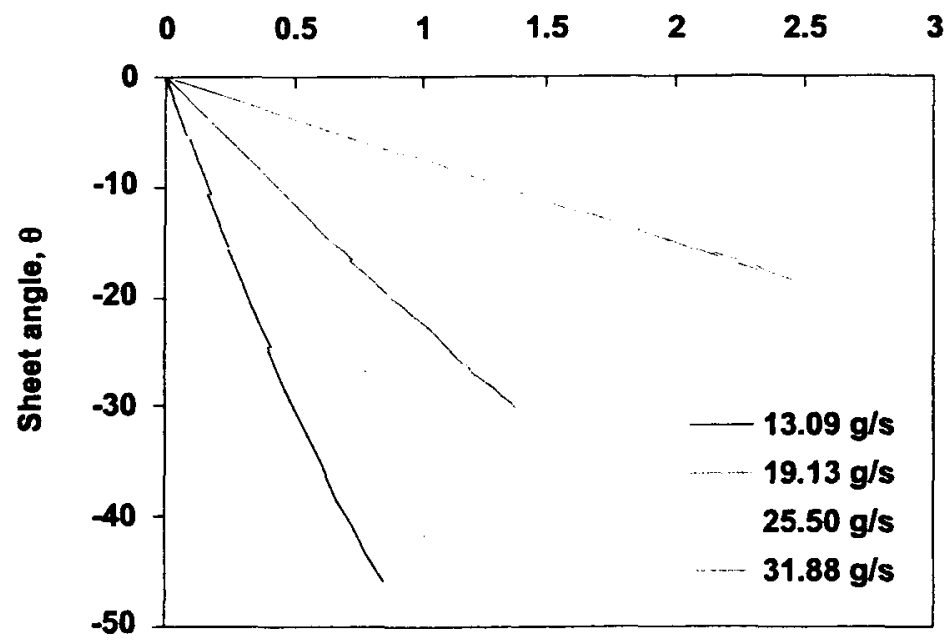

Figure 3. Axial variation of angle for a non-swirling liquid sheet.

In Fig. 4, the predicted variations of dimensionless sheet thickness, $\delta / \delta_{0}$, are plotted as functions of dimensionless axial distance. Figure 4 indicates that, at a given flow rate, the sheet thickness increases in the downstream direction. The sheet thickening is enhanced at higher liquid flow rates. The increase in sheet thickness in the axial direction is due to the contraction of the annular sheet as observed in Figs. 2 
and 3. The observations in Figs. 2, 3 and 4 are in general agreement with those of Baird and Davidson ${ }^{19}$ and Chuech and co-workers. ${ }^{13-16}$

The results in Fig. 5 reveal that the dimensionless sheet stream-wise velocity exhibits a complex behavior. At small liquid flow rates, the stream-wise velocity first increases nearby the nozzle orifice and then decreases further downstream. However, at higher flow rate, the stream-wise velocity experiences a monotonic reduction in the axial direction. The behavior of stream-wise velocity may be explained in light of mass conservation. At small flow rates, the initial rate of reduction in sheet radius is greater than thickness enlargement and therefore the velocity must increase to conserve mass. At larger distances from the nozzle, the rate of increase in sheet thickness picks up and exceeds that of radius reduction dictating a decrease in velocity for mass to be conserved. At higher flow rates, the decrease in sheet radius always outweighs the increase in its thickness causing the stream-wise velocity to be continuously reduced downstream. Figure 5 also shows that the dimensionless velocity decreases with an increase in mass flow rate. However, it should be noted that the velocity is made dimensionless by dividing by its initial value, $u_{f 0}$, which increases with mass flow rate as calculated from Eqs. (16) and (17) and listed in Fig. 5. Therefore, the magnitude of the dimensional velocity actually increases with mass flow rate as expected.

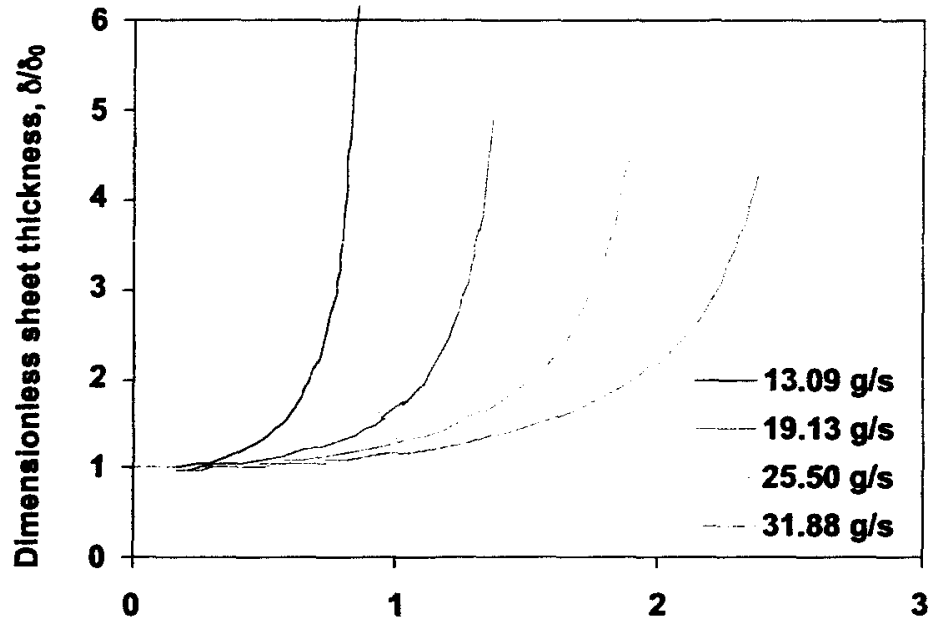

Dimensionless axial distance, $z / D_{0}$

Figure 4. Axial variation of dimensionless thickness for a non-swirling liquid sheet.

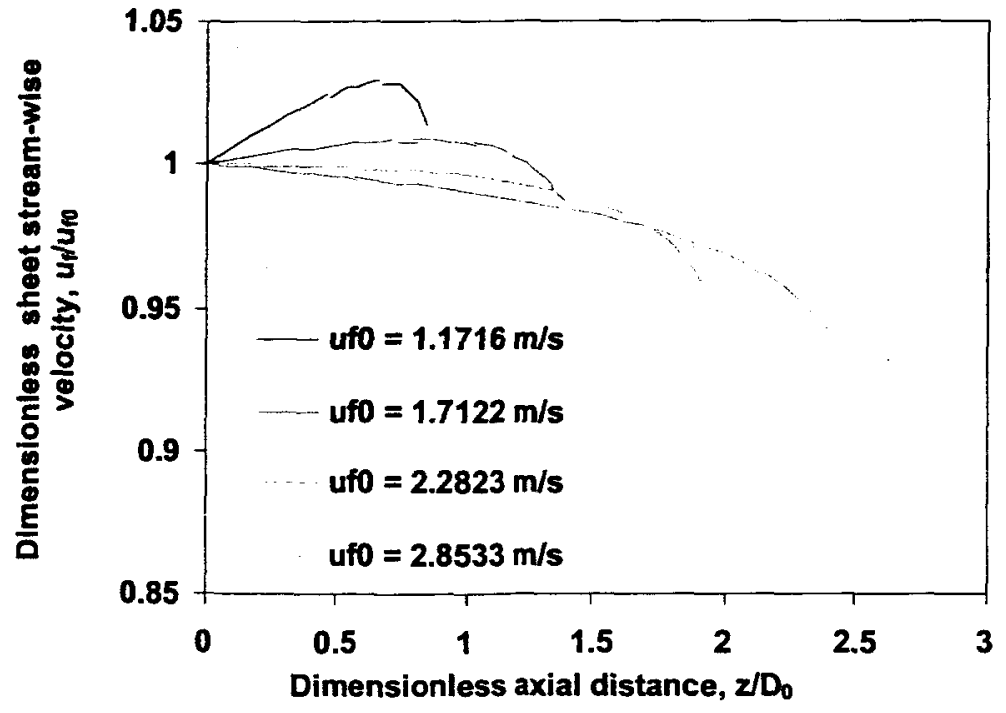

Figure 5. Axial variation of stream-wise velocity for a non-swirling liquid sheet. 
Chuech and co-workers' predictions indicated a monotonic increase in stream-wise velocities with axial distance at all flow rates investigated. The discrepancy between the present results and Chuech and co-workers' is believed to be due the neglect of interfacial friction effects in their computations, as alluded to earlier. Note that interfacial friction has a decelerating influence on the liquid sheet that increases with mass flow rate. The gravitational forces have the opposite role of accelerating the flow. Therefore, at a low mass flow rate, the interfacial friction is small and gravity dominates inducing a net acceleration. The opposite is true at higher mass flow rates.

Predicted cross sectional profiles of the liquid sheet at four different flow rates are shown in Fig. 6. The color codes on the annular sheet cross-sections represent variations of sheet velocities in the streamwise $\xi$-direction. Upon emerging from the annular nozzle orifice the predicted cross-sectional streamlines of the water sheet continuously converge toward the centerline resulting in the formation of a bell-shaped annular sheet that ultimately collapses into a round jet. The bell shape variations in Fig. 6 indicate that as the liquid flow rate is increased, the convergence rate of the liquid sheets decrease producing a more elongated bell with larger radius but smaller curvature. This is due to the greater inertia forces created at an increased mass flow rate which act to reduce contraction effects of surface tension forces. The results in Fig. 6 indicate that the stream-wise sheet velocities downstream are always less than their initial values at the nozzle orifice.

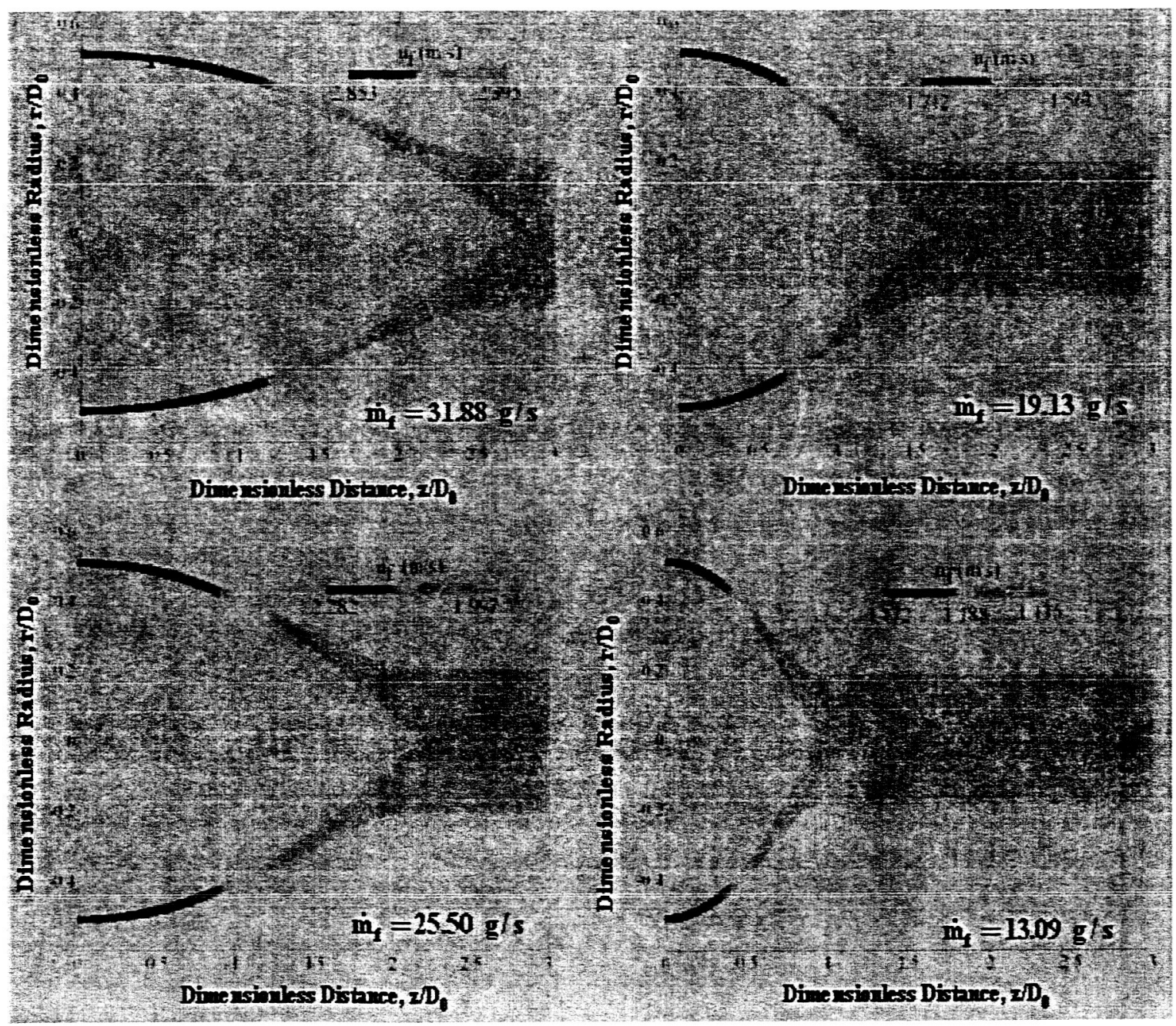

Figure 6. Influence of mass flow rate $f$ on the features of a non-swirling liquid sheet. 


\section{SWIRLING SHEET}

In this section, the swirl effects are included and hence the centrifugal and Coriolis forces are accounted for. The flow conditions are taken to be similar to those used by Chuech and co-workers ${ }^{13-16}$ to permit direct reference to their results. Therefore, the annular sheet diameter at the nozzle orifice is $D_{0}=$ $6.63 \mathrm{~mm}$. The initial sheet thickness is taken to be, $\delta_{0}=0.1524 \mathrm{~mm}$, which is equivalent to $1 / 4$ of the prefilmer width and is considered to be invariable with other flow conditions. To study the effect of liquid-port angle, or liquid-swirler angle, $\alpha$, on the results, this angle is varied in the calculations between $0^{\circ}$ and $60^{\circ}$. The liquid mass flow rates, $f$, under consideration are $17.76,40.82$, and $79.13 \mathrm{~g} / \mathrm{s}$. The liquid fuel properties include density, $\rho_{f}=765 \mathrm{~kg} / \mathrm{m}^{3}$, dynamic viscosity, $\mu_{4}=9.2 \times 10^{4} \mathrm{~kg} / \mathrm{m} . \mathrm{s}$, and surface tension, $\sigma=$ $0.025 \mathrm{~N} / \mathrm{m}$. The surrounding gas is assumed to be air at atmospheric conditions with density of $\rho_{g}=1.22$ $\mathrm{kg} / \mathrm{m}^{3}$ and dynamic viscosity of $\mu_{g}=17.9 \times 10^{-6} \mathrm{~kg} / \mathrm{m} . \mathrm{s}$.

The effects of liquid-swirler angle on the shape of the swirling liquid sheet are first examined. Figures 7 and 8 portray variations of sheet radius and angle at a constant liquid mass flow rate of $f=$ $17.76 \mathrm{~g} / \mathrm{s}$ and initial sheet angle of $\theta_{0}=0^{\circ}$, for liquid-swirler angles of $\alpha=0^{\circ}, 5^{\circ}, 10^{\circ}, 20^{\circ}, 30^{\circ}, 45^{\circ}$, and $60^{\circ}$. It is seen in Figs. 7 and 8 that, except at $\alpha=0^{\circ}$, both the radius and angle of the sheet are increased with the liquid-swirler angle. While the sheet radius exhibits a monotonic increase in the axial direction, the angle experiences an initial increase followed by a gradual decrease with the axial distance from the nozzle. The case when $\alpha=0^{\circ}$ corresponds to non-swirling sheet where the sheet converges towards its centerline and its radius decreases while the absolute values of the negative sheet angle increase with axial distance, as has been discussed earlier in relation with Figs. 2 and 3 . Therefore, the annular sheet is transformed from a converging bell to a diverging hollow-cone as a result of introducing swirt in the liquid. Swirling the liquid gives rise to centrifugal and Coriolis forces which act against the contracting effects of surface tension forces causing the sheet to move outward in the radial direction. It is evident from Figs. 7 and 8 that the present model is capable of smoothly predicting the transition from a bell-shaped non-swirling sheet to a swirling hollow-cone sheet in agreement with previous studies. ${ }^{13-16}$

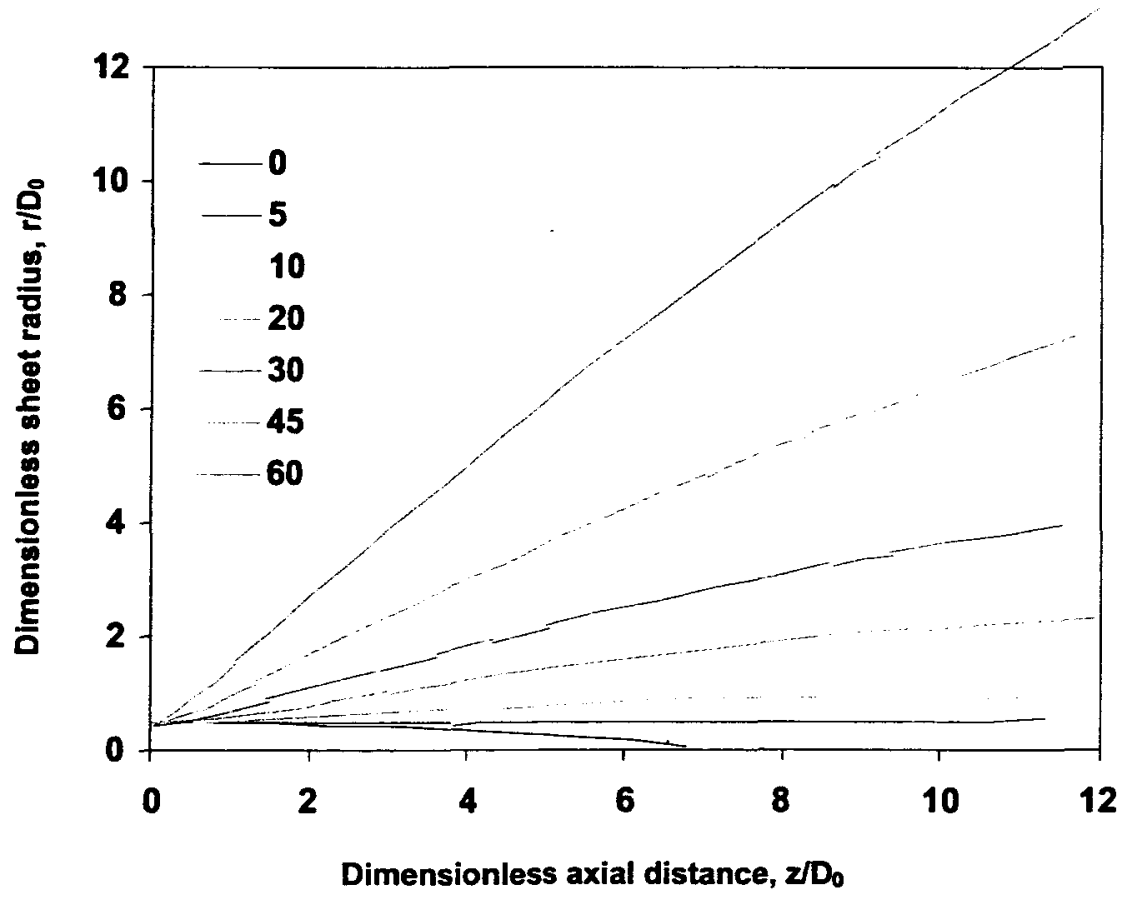

Figure 7. Axial variation of radius with liquid-swirler angle for a swirling sheet.

Figure 9 displays the dimensionless sheet thickness variation versus dimensionless axial distance from the nozzle exit for the same parameters considered in Figs. 7 and 8 . It is noted that except for a nonswirling sheet of $\alpha=0^{\circ}$, the sheet thickness continuously decrease as a consequence of the increase in sheet radius to satisfy mass conservation. Since the sheet radius decreases at a liquid-swirler angle in the absence of swirl, its thickness increases as indicated earlier with regards to Fig. 4. Chuech and co- 
workers $^{13-16}$ didn't investigae the dependence of liquid-swirler angle on a swirling hollow-cone sheet thickness.

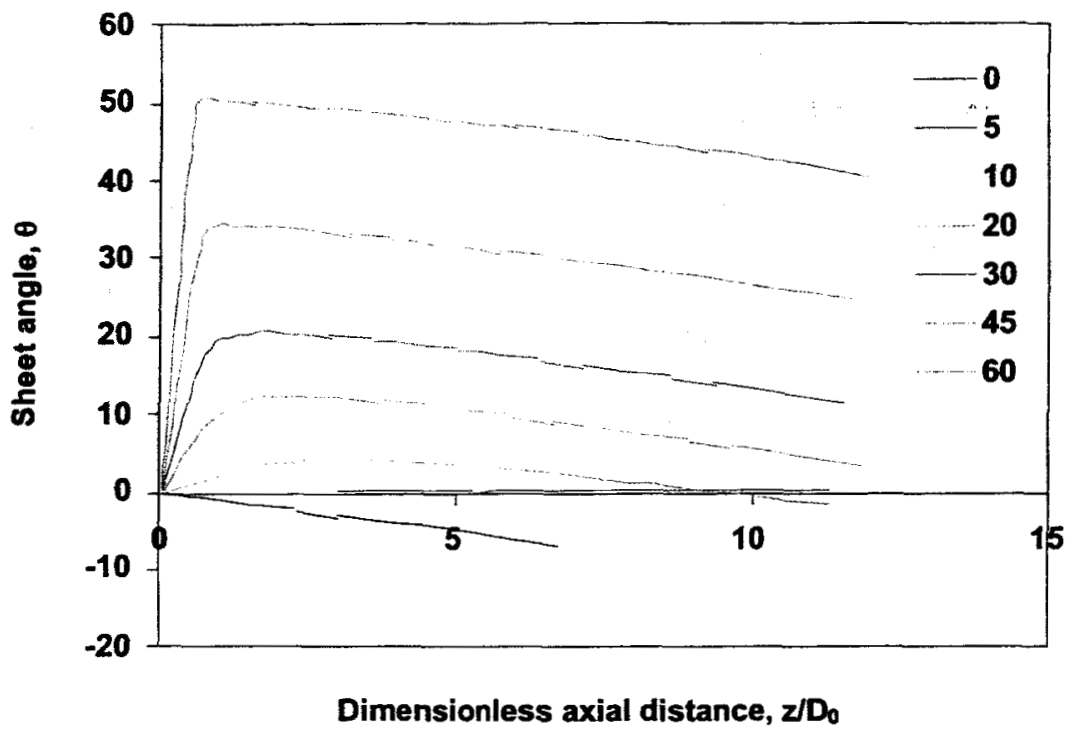

Figure 8. Axial variation of angle with liquid-swirler angle for a swirling sheet.

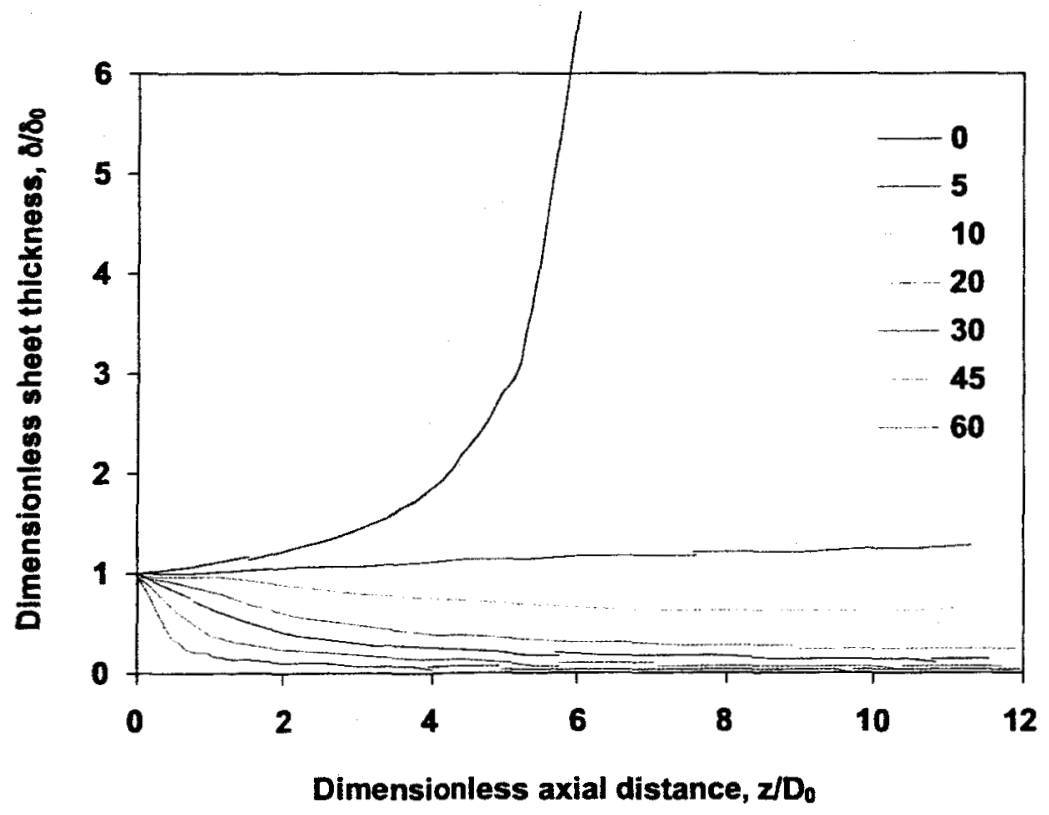

Figure 9. Axial variation of thickness with liquid-swirler angle for a swirling sheet.

Figures 10 and 11 portray the variations of the dimensionless stream-wise and swirl (tangential) velocity components against the dimensionless axial distance at the same flow conditions of Figs. 7, 8 and 9. At small liquid-swirler angles close to $0^{\circ}$, the stream-wise velocity continuously decreases with distance in the axial direction. At larger liquid-swirler angles, there is an initial increase in the stream-wise velocity proceeded by a gradual decrease. The initial increase in the stream-wise velocity at small liquid-swirler angles correspond to the sharp decrease in the sheet thickness observed in Fig. 9. Therefore, it is concluded that the behavior of the stream-wise velocity is consistent with mass conservation. As seen 
earlier in Fig. 8, the sheet angle also possesses the same type of behavior as the stream-wise velocity. It is envisaged that this behavior is due to the steeper increase in sheet radius as it moves far from the nozzle which causes the decelerating effects of interfacial friction and surface tension forces to overpower the accelerating actions of the centrifugal and gravitational forces producing reduction in sheet curvature and stream-wise velocity. Recall that the stream-wise velocity of a non-swirling sheet also exhibited a simiar behavior in connection with the mass flow rate as delineated in Fig. 5. The dimensionless tangential velocity aiways decreases with the axial distance from the nozzle as the liquid-swirler angle is increased. Note that at $\alpha=0$ the swirl velocity $w_{f}$ vanishes. It is important to keep in mind that the dimensionless swirl velocity is normalized by its initial value at the nozzle exit which increases as $\tan (\alpha)$ for a constant mass flow rate as given by Eqs. (16) and (17). This means that the magnitudes of the dimensional swirl velocities are smaller than they appear in Fig. 11 for $\alpha<45^{\circ}$ and larger for $\alpha \geq 45^{\circ}$. Knowledge of stream-wise and tangential velocities at the locations of liquid sheet rupture is needed to resolve the resultant ligament and drop trajectories as well as drop formation rate. Chuech and co-workers ${ }^{13-16}$ didn't present results for the influence of liquid-swirler angle on stream-wise and tangential velocities of a hollow-cone sheet.

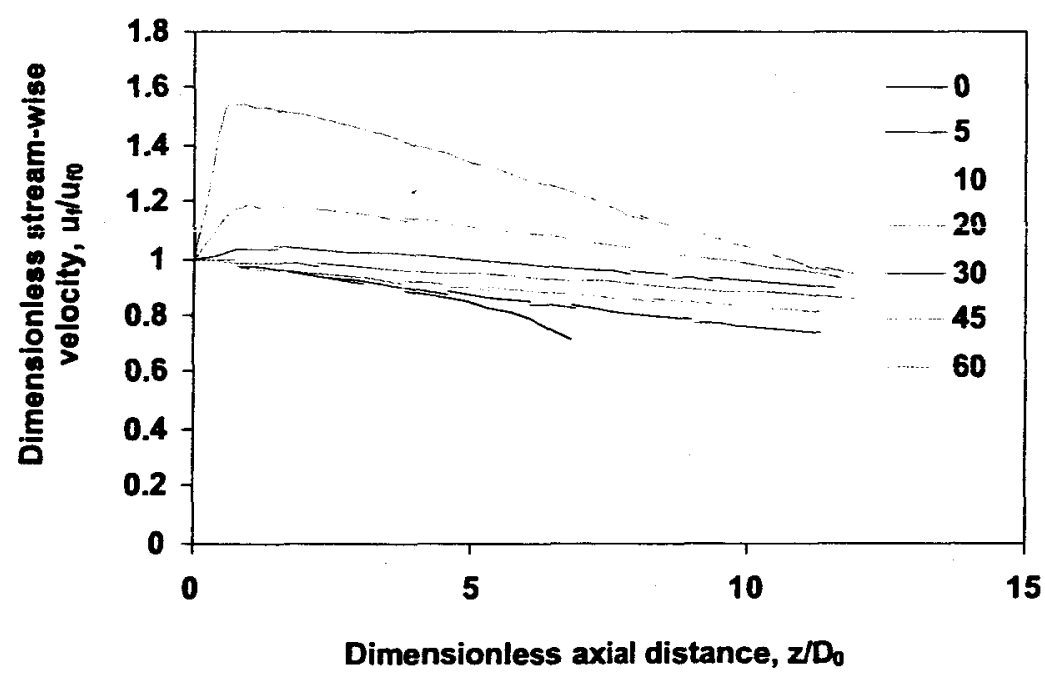

Figure 10. Axial variation of stream-wise velocity for a swirling liquid sheet.

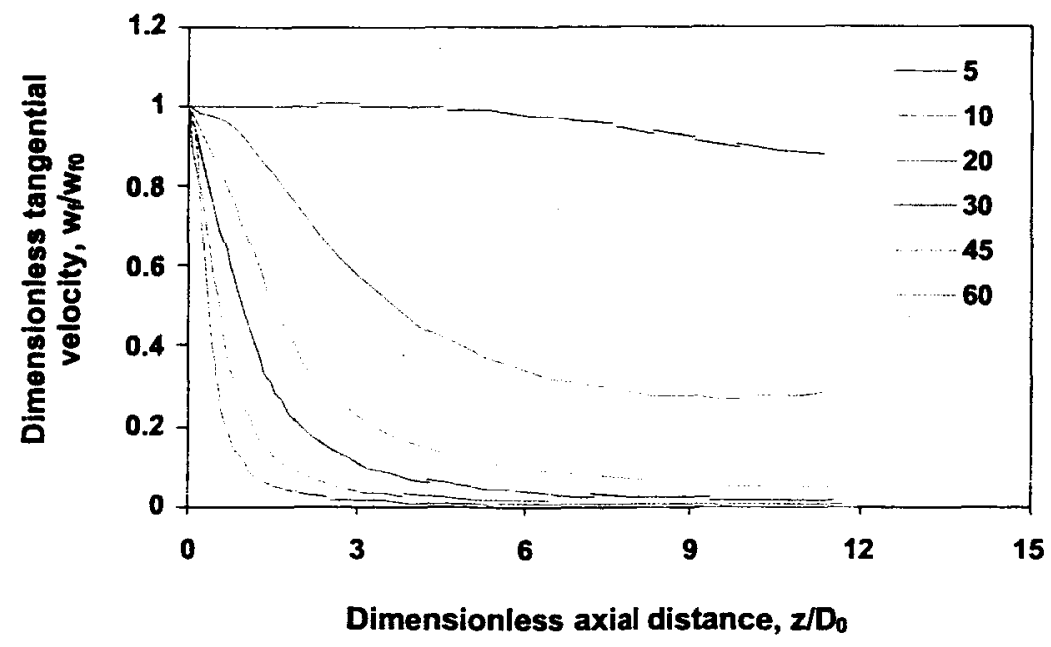

Figure 11. Axial variation of tangential velocity for a swirling liquid sheet. 
To investigate the influence of mass flow rate on the swilling hollow-cone sheet characteristic, the variations of the sheet angle with axial distance from the nozzle at liquid mass flow rates of 17.76, 40.82, and $79.13 \mathrm{~g} / \mathrm{s}$ and an initial sheet injection angle $\theta=0^{\circ}$ for a liquid-swirler angle of $\alpha=30^{\circ}$ are depicted in Fig. 12. The sheet angle increases with the mass flow rate due to higher inertia and centrifugal forces that overcome the opposing effects of liquid contraction by surface tension and deceleration due to interfacial friction. Initially, as the sheet emerges from the nozzle its angle rises sharply over a short axial distance then remains aimost constant further downstream. However, it is noted that at the lowest mass flow rate the sheet tends to curve toward the centerline since the inertia and centrifugal forces become weaker than surface tension and gravitational forces. Predictions of sheet angles are of fundamental significance in spray applications since the maximum sheet angles determine the spray angle and consequently the orientation of the drops formed by sheet atomization.

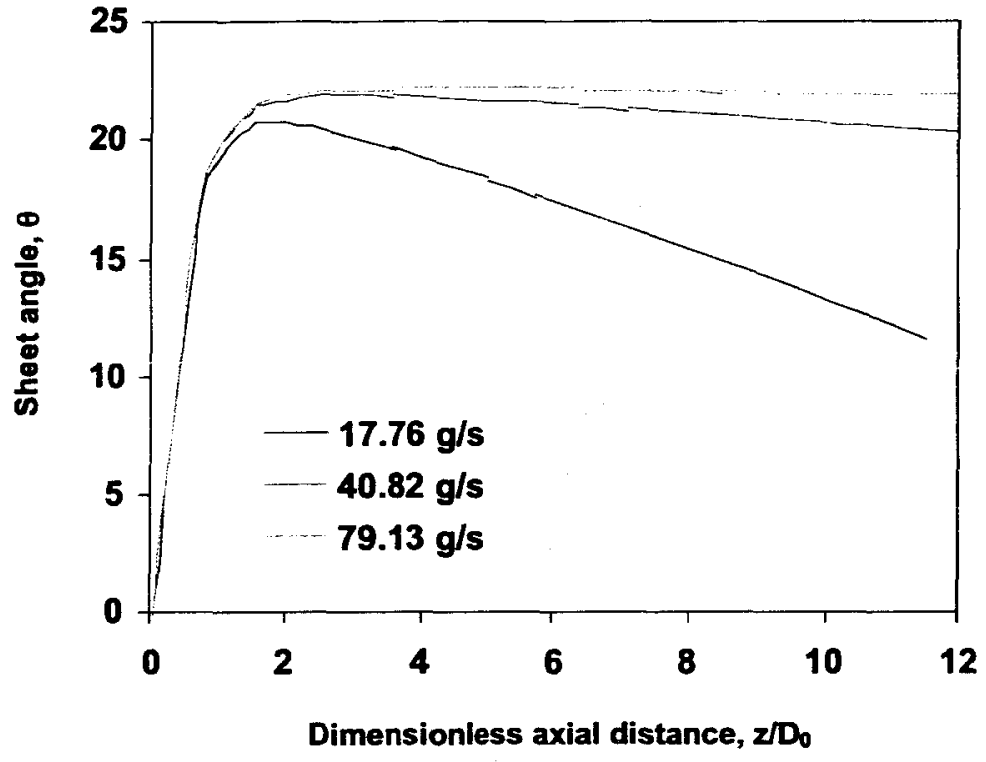

Figure 12. Axial variation of angle with mass flow rate for a swirling liquid sheet.

The variations of the dimensionless sheet thickness with dimensionless axial distance are displayed in Fig. 13 for the same flow conditions considered in Fig. 12. It is observed that the sheet becomes thinner in the axial direction due to the expected expansion of its surface area by the action of centrifugal forces as will be elucidated later. The thinning of the sheet is slightly enhanced as the liquid mass flow rate is increased. Recall that the non-swirfing sheet thickness increased dramatically with the axial distance and mass flow rate as viewed in Fig. 4. It is well known ${ }^{20}$ that the film thickness along the sheet trajectory is detrimental to the drop sizes that are produced by sheet disintegration. Therefore, it may be concluded based on the present work that using a higher fuel mass flow rate and fuel-swirler angle would produce smaller drops, a trait that is highly desirable in fuel atomization applications.

Chuech and co-workers ${ }^{13-16}$ presented their results in terms of fuel pressure drop but didn't provide the nozzle discharge coefficient to allow for determining the corresponding mass flow rate. Also, their computations neglected the interfacial friction effects given by Eqs. (6) and (7) and hence are only applicable to an annular liquid sheet in a vacuum surrounding. This situation renders a quantitative comparison with Chuech and co-workers rather difficult. However, the general trends of the present results for mass flow rate variations are similar to Chuech and co-workers' results for fuel pressure drop variations.

Figure 14 portrays cross-sectional profiles of a swirling hollow-cone sheet at four liquid mass flow rates, $13.09,19.13,25.5$, and $31.88 \mathrm{~g} / \mathrm{s}$. It is evident that including the swirl effect causes the liquid sheet to be transformed from an annular bell-shape to a hollow-cone configuration. Increasing the mass flow rate enhances the sheet's "opening-up" in the axial direction as a result of increased sheet radius and angle. The color codes across the sheet thickness delineate the change of stream-wise velocity. The sheet slows down as it moves away from the nozzle due to the increase in its radius. The magnitude of the stream-wise velocity is larger at higher liquid flow rates. As can be see from Fig. 14, the sheet stream-wise velocity only varies slightly with mass flow rate. Although not reported here for brevity, the sheet tangential velocity was 
also found to possess little dependence on mass flow rate. These observations are similar to Chuech and co-workers'.

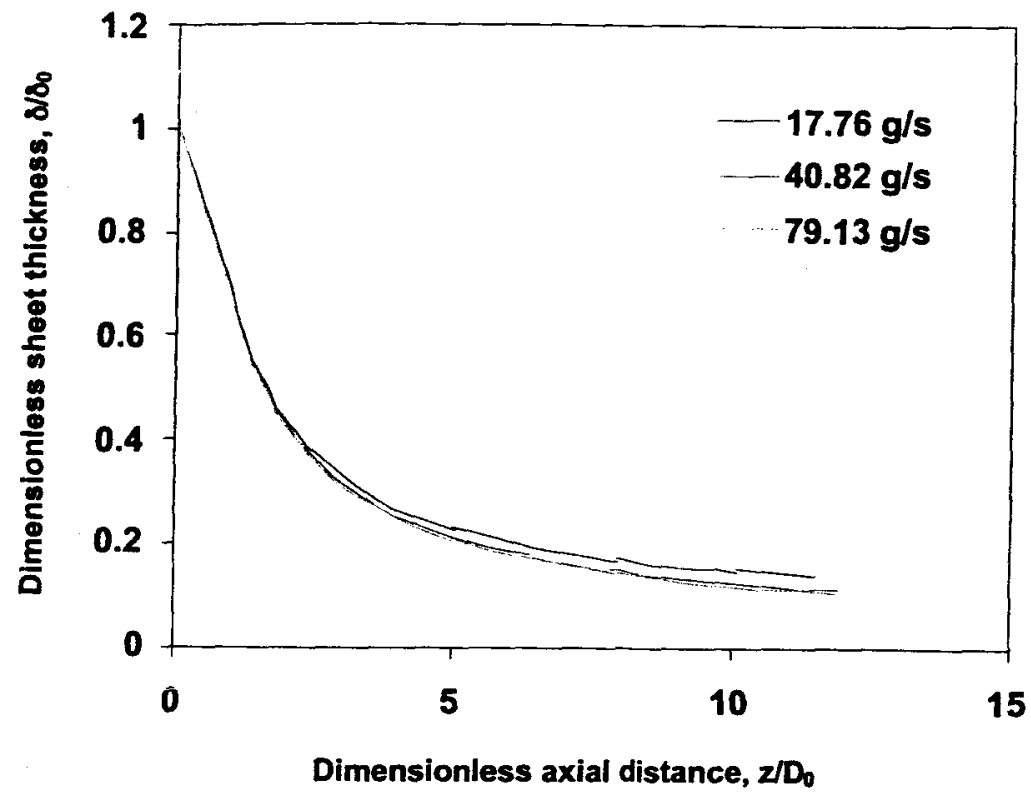

Figure 13. Axial variation of thickness with mass flow rate for a swirling sheet.

\section{SUMMARY AND CONCLUSIONS}

A mathematical model that utilizes body-fitted curvilinear coordinates to simplify the analyses of non-swirling and swirling liquid sheets issued from an annular nozzle in a quiescent surrounding medium has been formulated and presented in a clear and organized manner. An adequate description of the numerical approach used to solve the system of nonlinear first-order differential equations involved is provided for effortless adoption of the model by interested researchers. Solutions of the model's governing equations yield sheet trajectory, angle, thickness and velocity at different flow parameters for both the nonswirling and swirling cases.

The results indicate that when no swirl is present in the liquid, a bell-shaped annular sheet is formed. Bell elongation and curvature increase with liquid mass flow rate due to the higher inertia forces which pre-dominate the contracting effects of surface tension. As the bell-shaped annular sheet converges to its centerline, the sheet radius decreases and its thickness increases in the downstream direction as a direct consequence of mass conservation. At high flow rates, the stream-wise velocity decreases monotonically in the axial direction while it shows an initial increase followed by a decrease at lower flow rates. A full round jet is ultimately formed when the bell coalesces at the centerline.

For a swirling liquid sheet, the centrifugal cause the sheet to diverge in the axial direction assuming a hollow-cone configuration. The sheet radius increases and its thickness decreases in the axial direction when the liquid-swirter angle or mass flow rate is increased due to the dominance of inertial and centrifugal forces over capillary and interfacial friction forces. Both the sheet angle and stream-wise velocity experience an initial increase followed by a decrease in the axial direction at larger liquid-swirler angle or mass flow rates. The swirt velocity is decreased in the axial direction as the liquid-swirter angle is increased. 


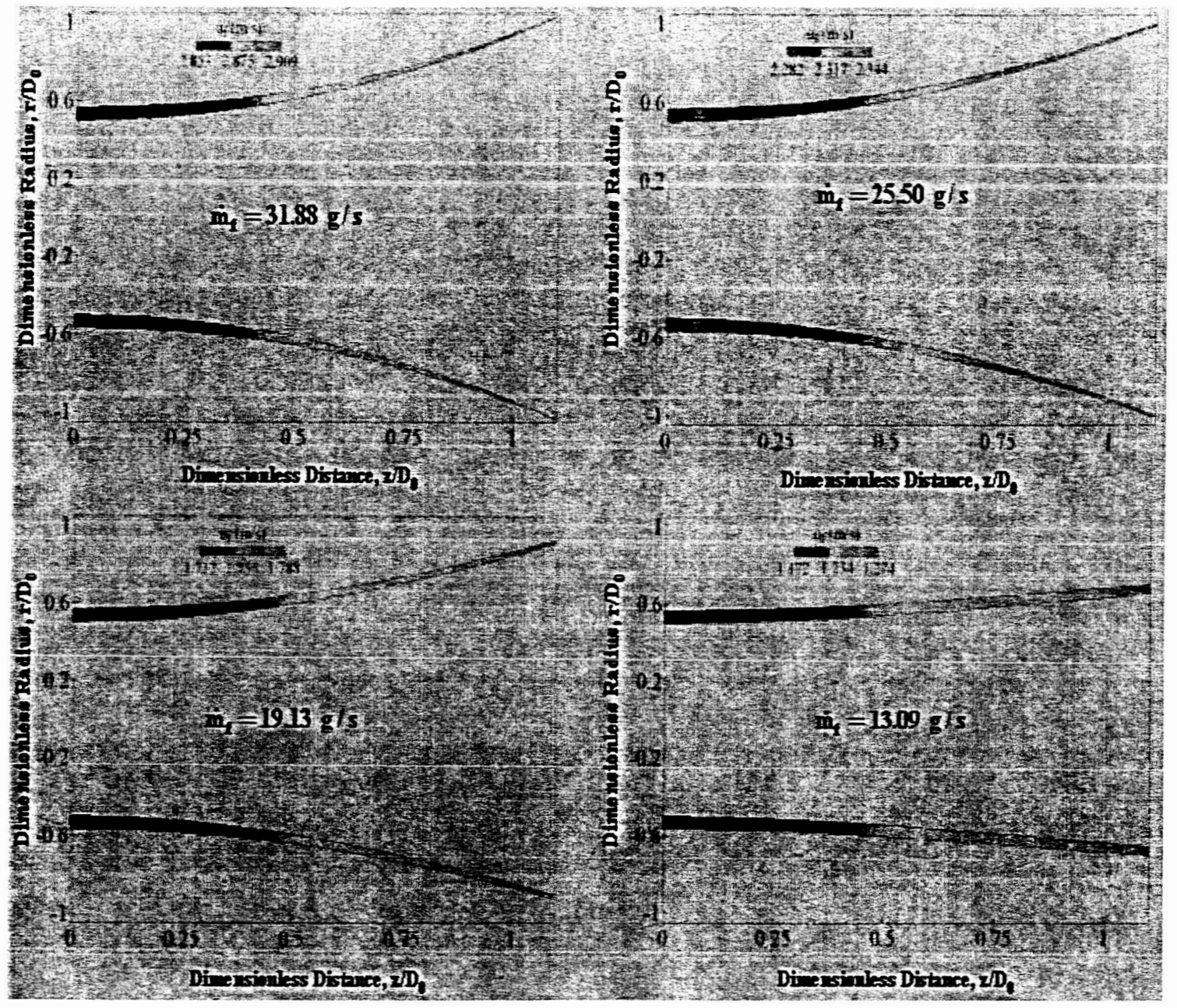

Figure 14. Influence of mass flow rate on the features of a swirling liquid sheet.

\section{FUTURE WORK}

Validation of the model predictions against experimental data and empirical correlations are planned. The present model may be incorporated in computational codes used to simulate injection performance of swirl atomizers. The model is capable of predicting the characteristics of the sheet produced from the atomizer such as its trajectory, thickness and velocity. These parameters are prerequisites for a realistic account of spray behavior in combustion simulations. The model may be supplemented with a sheet breakup module to effect predictions of atomization characteristics such as breakup length and thickness as well as drop size, velocity and orientation. Recently, sheet breakup models ${ }^{21,22}$ that are based on the nonlinear evolution of Kelvin-Helmholtz instability waves have been developed and proved to be more practical than linearized breakup models. However, these nonlinear breakup models are successful only if accurate predictions of the sheet initial flow parameters are provided. These parameters are highly dependent on nozzle geometry and internal flow conditions which may be investigated using ad hoc and commercial CFD codes. ${ }^{23-25}$

\section{REFERENCES}

1. Feikema, D. A., Eskridge, R., and Hutt, J., Structure of a Non-Evaporating Swirl Injector Spray, Atomization and Sprays, Vol. 7, No. 1, pp. 77-95 (1997). 
2. Stephens, J. R., Acharaya, S., Gutmark, E. J., An Experimental Study: Swirl-Stabilized Spray Combustion with Active Forcing, AlAA Paper 99-0330, $37^{\text {th }}$ Aerospace Sciences Meeting, Reno, NV, Jan11-14, 1999.

3. Schmidt, D. P., Chiapetta, L. M., Goldin, G. M., and Madabhushi, R. K., Transient Multidimensional Modeling of Air-Blast Atomizers, Atomization and Sprays, Vol. 13, No. 4, pp. 373-394 (2003).

4. Han, Z., Parrish, S., Farrell, P. V., and Reitz, R. D., Modeling Atomization Processes of PressureSwirl Hollow-Cone Fuel Sprays, Atomization and Sprays, Voi. 7, No. 6, pp. 663-684, (1997).

5. York, J. L., Stubbs, H. E., and Tek, M. R., The Mechanism of Disintegration of Liquid Sheets, Transactions of ASME, Vol. 75, pp. 1279-1286 (1953).

6. Dombrowski, N., and Johns, W. R., The Aerodynamic Instability and Disintegration of Viscous Liquid Sheets, Chemical Engineering Science, Vol. 18, pp. 203-214 (1963).

7. Senecal, P. K., Schmidt, D. P., Nouar, I., Rutland, C. J., and Reit, R. D., Modeling High Speed Viscous Liquid Sheet Atomization, International J. of Multiphase Flow, Vol. 25, pp. 1073-1097 (1999).

8. Weber, C., Zum Zerfall eines Flussigkeitsstrahle (On the Breakdown of a Fluid Jet), Z. Angew. Math. Mech., Vol. 11, pp. 136-159 (1931).

9. Kawano, S., Hashimto, H. Togari, H., Ihara, A., Suzuki, T., and Harada, T., Deformation and Breakup of an Annular Liquid Sheet in a Gas Stream, Atomization and Sprays, Vol. 7, No. 4, pp. $359-374$ (1997).

10. Conto, H-S., Carvalho, Jr., , J. A., and Bastos-Netto, D., Theoretical Formulation for Sauter Mean Diameter of Pressure-Swirl Atomizers, J. Propulsion and Power, Vol. 13, pp. 691-696 (1997).

11. Lee, J. G., and Chen, L. D., Linear Stability Analysis of Gas-Liquid Interface, AlAA J., Vol. 29, pp. 1589-1595 (1991).

12. Panchagnula, M. V., Sojka, P. E., Santangelo, P. J., On the Three Dimensional Instability of a Swirling Annular Inviscid Liquid Sheet Subject to Unequal Gas Velocities, Physics of Fluids, Vol. 8, No. 12, pp. 3300-3312 (1996).

13. Mao, C. P., Chuech, S. G., and Przekwas, A. J., An Analysis of Pressure Swirl and Pure Airblast Atomization, Atomization and Sprays, Vol. 1, No. 2, p.p. 215-235 (1991).

14. Chuech, S. G., Numerical Simulation of Nonswirling and Swirling Annular Liquid Jets, AlAA Paper 92-0464, 30 ${ }^{\text {th }}$ Aerospace Sciences Meeting, Reno, NV, January 6-9, 1992.

15. Chuech, S. G., Numerical Simulation of Nonswirling and Swirling Annular Liquid Jets, AlAA Joumal, Vol. 31, No. 6, p.p. 1022-1027 (June 1993).

16. Przekwas, A. J., Theoretical Modeling of Liquid Jet and Sheet Breakup Process," Recent Advances in Spray Combustion: Spray Atomization and Drop Burning Phenomena, AIAA Inc., Vol. 1, pp.211-239, (1996).

17. Mongia, H. C., Reynolds, R. S., and Srinivasan, R., Multidimensional Gas Turbine Combustion Modeling: Applications and Limitations, AIAA J., Vol. 24, No. 6, pp. 890-904 (1986).

18. Rizk, N. K., and Lefebvre, A. H., The Influence of Liquid Film Thickness on Air Blast Atomization, ASME J. of Engineering for Power, Vol. 102, No. 7, p.p. 706-710 (July 1980).

19. Baird, M. H. I., and Davidson, J. F., Annular Jets-I, Chemical Engineering Science, Vol. 17, No. 10, pp. 467-472 (1962).

20. Lefebvre, A. H., Atomization and Sprays, Hemisphere, New York, pp. 173-181 (1989).

21. Clark, C. J., and Dombrowski, N., Aerodynamic Instability and Disintegration of Inviscid Liquid Sheets, Proceedings of the Royal Society of London, Vol. A329, pp. $467-478$ (1972).

22. Ibrahim, E. A., Characteristics of the Spray Produced by Liquid Sheet Atomization, J. of Turbo and Jet Engines, Vol. 14, pp. 159-171 (1997).

23. Liao, Y., Sakman, A. T., Jeng, S. M., Jog, M. A., and Benjamin, M. A., A Comprehensive Model to Predict Simplex Atomizer Performance, J. Engineering for Gas Turbines and Power, Vol. 121, pp. 285294 (April 1999).

24. Jang, C. S., Bae, C. S., and Choi, S. M., Characteristics of Prototype High-Pressure Swirl Injector Nozzles, Part II: CFD Evaluation of Internal Flow, Atomization and Sprays, Vol. 10, No. 2, pp. $179-197$ (2000).

25. Yule, A. J., and Chinn, J. J., The Internal Flow and Exit Conditions of Pressure Swirl Atomizaers, Atomization and Sprays, Vol. 10, No. 2, pp. 121- 146 (2000). 